\title{
L'AVC du sujet âgé : vers une prise en charge spécialisée à la phase aiguë et en SSR ?
}

\section{Stroke in the elderly: toward a specialized care in acute and rehabilitation units?}

\author{
Y. Guilhermet • L. Valdelièvre • P. Krolak-Salmon \\ Reçu le 3 mars 2010 ; accepté le 13 avril 2010 \\ (C) Springer-Verlag France 2010
}

Résumé Longtemps, l'âge avancé des patients a été un critère discriminant pour les accueillir dans des unités neurovasculaires et de rééducation spécialisées dans la prise en charge de l'AVC. Il est depuis démontré que cette prise en charge diminue la mortalité, le nombre d'entrées en institution, augmente la capacité de récupération fonctionnelle, la qualité de vie et donc les possibilités de retour au domicile, et ainsi la qualité de vie. Elle est d'autant plus efficace, qu'elle s'inscrit dans une filière rassemblant les unités de soins de courte durée (SCD) neurologiques et/ou gériatriques et les unités de soins de suite et réadaptation (SSR). L'expérience de l'unité de SSR de la filière AVC de la personne âgée de l'hôpital des Charpennes (hospices civils de Lyon) permet de montrer, grâce à des indicateurs de suivi précis, que la prise en charge spécialisée et globale de ces patients apporte des bénéfices importants.

Mots clés Unité neurovasculaire · Unité de soins de suite et réadaptation - Personne âgée

\begin{abstract}
Patients presenting with a stroke may be refused by neurological rehabilitation center teams because of their old age. However, it has been disclosed that stroke units and specialized rehabilitation centers are efficient in terms of mortality, functional recovering, quality of life, and home return regardless the age. The experience of the rehabilitation unit of the Charpennes Hospital in Lyon, dedicated to old patients presenting with a stroke, is of particular interest to demonstrate the feasibility and the efficiency of such a specialized care in elderly population. Combined acute
\end{abstract}

Y. Guilhermet $(\bowtie) \cdot$ L. Valdelièvre $\cdot$ P. Krolak-Salmon Hôpital des Charpennes, CHU de Lyon, 27, rue Gabriel-Péri, F-69100 Villeurbanne, France

e-mail : yves.guilhermet@chu-lyon.fr

L. Valdelièvre

Inserm U 821, université Claude-Bernard, Lyon-I, F-69373 Lyon, France and rehabilitation stage stroke units may be particularly appropriated to elderly.

Keywords Stroke unit · Rehabilitation center · Elderly

\section{Introduction}

Responsable de 5 à $7 \%$ du coût de la santé en France, l'accident vasculaire cérébral (AVC) représente un problème de santé publique. La prévalence des AVC ischémiques et hémorragiques est de 40/1000 au-delà de 80 ans. On enregistre environ 80 à 100000 nouveaux cas par an. C'est la première cause de handicap liée au vieillissement, la troisième cause de démence et la troisième cause de mortalité dans les pays développés [1]. Les conséquences d'une telle pathologie sont multiples, médicales, sociales et économiques. La fréquence des AVC augmente avec l'âge des patients, plus de la moitié des hémiplégiques ont plus de 75 ans [2]. Le décès précoce (avant 30 jours) survient dans $20 \%$ des cas.

Il est démontré que les soins ne peuvent atteindre leur pleine efficacité, que s'ils s'inscrivent dans une filière structurée [3]. Or, la filière AVC est insuffisante, en particulier pour les personnes âgées, comme le montre notamment l'étude d'observation des délais de prise en charge des AVC dans le département du Rhône [4]. Sur 1226 cas d'AVC chez des patients d'âge moyen de 72,4 ans, seulement $43 \%$ ont été régulés par le SAMU à la phase préhospitalière. Quatre-vingt-un pour cent des patients ont été admis aux urgences, $5 \%$ en réanimation et seulement $9 \%$ en unité neurovasculaire (UNV), contre $60 \%$ en Norvège, $36 \%$ en Angleterre et $30 \%$ en Allemagne pour un âge comparable. Un transfert en UNV a été demandé pour $26 \%$ des patients. Il a été refusé pour $32 \%$ d'entre eux soit par manque de place (48\%), soit pour nonindication selon le délai ou l'âge (48\%). L'insuffisance du nombre de structures spécialisées en médecine physique et de rééducation peut induire une discrimination sur le simple critère d'âge des patients [5]. 
Or, la rééducation précoce, durable et intense a montré son efficacité chez la personne âgée. Cette dernière nécessite une prise en charge spécialisée et globale, prenant en compte sa fragilité potentielle, ses comorbidités, son statut cognitif notamment. Il est nécessaire d'analyser les variables liées à l'état antérieur du patient (âge, historique du patient, situation sociofamiliale, autonomie avant l'AVC, état cognitif) et celles liées à l'AVC (gravité du déficit neurologique, déficiences, incapacités), afin de déterminer le type de rééducation et d'élaborer un projet de vie adapté. Cela nécessite une évaluation pluridisciplinaire [6-11]. L'AVC chez le sujet âgé n'est pas synonyme de soins palliatifs ou de soins de longue durée. L'âge, les troubles cognitifs, l'héminégligence, les troubles aphasiques, la dépression et la situation sociofamiliale n'influencent pas de façon indépendante le devenir fonctionnel du patient [11].

L'efficacité des UNV et USINV a été démontrée par rapport aux structures de soins non spécialisées [12]. Le taux de mortalité ou d'institutionnalisation est significativement diminué lorsque les patients sont admis en UNV par rapport à une unité non spécialisée (14 versus $30 \%$; $p<0,001$ ) [12]. Le taux de patients vivant avec un handicap peu sévère (Rankin $<3$ ) à un an est plus élevé après hospitalisation en UNV (66 versus $85 \%=p<0,001$ ) [12], le risque de décès est diminué de $18 \%$ à un an après un passage en UNV par rapport aux unités non spécialisées [13]. Une étude italienne [14] confirme ces résultats avec une baisse du risque de décès et de handicap à deux ans pour tous les âges et tout niveau de gravité. Il est suggéré que le bénéfice lié à de telles unités repose principalement sur l'efficacité d'une équipe médicale et paramédicale expérimentée et pluridisciplinaire à condition toutefois de s'adresser à des patients âgés sans limitation significative de leur autonomie avant l'AVC.

\section{Expérience de l'unité soins de suite et réadaptation (SSR) de la filière avec des Charpennes}

Une unité de dix lits de SSR gériatriques de l'hôpital des Charpennes est inscrite depuis 2003 dans la filière AVC du CHU de Lyon s'adressant aux patients de plus de 70 ans ayant présenté un AVC, en complément des services d'urgence, de neurologie et de médecine. Elle est animée par une équipe pluridisciplinaire unique au sein des hospices civils de Lyon, comportant notamment un gériatre, un médecin rééducateur, un neurologue, ainsi que des kinésithérapeutes, un ergothérapeute, un psychomotricien et un orthophoniste. Les statistiques (cf. Tableaux 1,2,3,4,5) portant sur les indicateurs généraux ont été réalisées sur la période 2003-2008. Celles portant sur des indicateurs plus spécifiques ont été réalisées pour l'année 2008, suite à la mise en place d'une base de données élargie.
Moyenne d'âge de la population accueillie (2003-2008)

L'âge moyen de la population accueillie est de 80,5 ans (61-98 ans) (Tableau 1).

\section{Taux de décès (2003-2008)}

Le taux moyen de décès sur la période 2003-2008 est de $5,6 \%$ (Tableau 2).

\section{Le devenir des patients (année 2008)}

Les résultats sont présentés en fonction de trois critères : lieu de vie initial, score de la mesure d'indépendance fonctionnelle (MIF) à l'admission et du déficit moteur à l'admission du patient.

\section{Lieu de vie des patients}

Sur les 66 patients admis en 2008, trois $(4,5 \%)$ vivaient en EHPAD (à définir) et $63(95,5 \%)$ vivaient à domicile. Sur les 63 patients vivant à domicile, quatre $(6,4 \%)$ furent mutés dans des unités de court séjour, neuf (14,3\%) furent admis en EHPAD, cinq (7,9\%) sont décédés, cinq $(7,9 \%)$ furent admis en soins de longue durée (SLD) et $40(63,5 \%)$ purent regagner leur domicile. Concernant les patients vivant en EHPAD, deux (66,6 \%) purent regagner leur EHPAD.

\section{Pourcentage de retour au domicile en fonction du score de la MIF à l'admission (Tableau 3)}

\section{Pourcentage de retour au domicile en fonction du déficit moteur à l'admission (Tableau 4)}

\section{Type d'AVC (année 2008)}

En 2008, 77,3 \% d'accidents ischémiques constitués (AIC) et $22,7 \%$ d'accident hémorragiques (AH) furent admis dans l'unité, ce qui est conforme aux données de la littérature chez la personne âgée.

\begin{tabular}{|llllll|}
\hline \multicolumn{4}{|c}{ Tableau $\mathbf{1}$} & \multicolumn{5}{c|}{ Moyenne d'âge de la population accueillie (2003-2008) } \\
\hline $\mathbf{2 0 0 3}$ & $\mathbf{2 0 0 4}$ & $\mathbf{2 0 0 5}$ & $\mathbf{2 0 0 6}$ & $\mathbf{2 0 0 7}$ & $\mathbf{2 0 0 8}$ \\
\hline 79 & 79,1 & 81,2 & 79,8 & 82,8 & 81,2 \\
\hline
\end{tabular}

\begin{tabular}{|lccccc|}
\hline \multicolumn{4}{|c}{ Tableau 2} & \multicolumn{5}{c|}{ Pourcentage de décès sur la période $2003-2008$} \\
\hline $\mathbf{2 0 0 3}$ & $\mathbf{2 0 0 4}$ & $\mathbf{2 0 0 5}$ & $\mathbf{2 0 0 6}$ & $\mathbf{2 0 0 7}$ & $\mathbf{2 0 0 8}$ \\
\hline $1,7 \%$ & $0,6 \%$ & $8,3 \%$ & $8,9 \%$ & $8 \%$ & $7,6 \%$ \\
\hline
\end{tabular}


Tableau 3 Pourcentage de retour au domicile en fonction du score de la MIF à l'admission

\section{Score de la MIF à l'admission Pourcentage de retour} au domicile (\%)

Groupe $1(\mathrm{MIF}<60) \quad 41$

Groupe $2(60<\mathrm{MIF}<80) \quad 70$

Groupe $3(\mathrm{MIF}>80)$

86

${ }^{\text {a }}$ Mesure d'indépendance fonctionnelle.

Tableau 4 Pourcentage de retour au domicile en fonction du déficit moteur à l'admission

\begin{tabular}{|ll|}
\hline Déficit moteur & $\begin{array}{l}\text { Taux de retour au } \\
\text { domicile }(\%)\end{array}$ \\
\hline Hémiplégie & 39,3 \\
Hémiparésie & 80 \\
\hline
\end{tabular}

\section{Profil cognitif et démence}

Le score de MMSE moyen est de 25,4/30 pour l'année 2008. Les profils cognitifs sont évocateurs dans la majorité des cas d'une maladie d'Alzheimer à composante cérébrovasculaire, et plus rarement d'une démence vasculaire liée à une lésion stratégique ou un état multilacunaire.

\section{Dépression}

Environ $40 \%$ des patients ont présenté, en 2008, une dépression dans les suites de leur AVC. Tous ces patients ont reçu un traitement antidépresseur. Il s'agit généralement d'un inhibiteur de la recapture de la sérotonine.

\section{Comorbidités (année 2008)}

Sur l'ensemble de la population accueillie en 2008, $43(65,2 \%)$ patients présentaient des comorbidités pouvant interférer dans la prise en charge rééducative (Tableau 5).

\section{Discussion}

\section{L'âge est-il un facteur de risque défavorable ?}

Calmels et al. soulignent " qu'un âge élevé, au-delà de 80 ans, est souvent discriminant dans la prise en charge ou dans l'orientation des patients, sans autre critère objectif apparent » [15]. De plus en plus d'études démontrent que l'âge des patients en soi n'est pas un facteur déterminant pour une récupération fonctionnelle après un AVC ; c'est plutôt l'état fonctionnel initial et la sévérité de l'AVC qui comptent [16,17]. Cela peut être appréhendé par une évaluation formalisée du pronostic fonctionnel en fonction de la MIF chez la personne âgée après un AVC, en comparaison avec une population plus jeune. La MIF fut développée en 1987 par Hamilton et al. [18] afin de mesurer l'indépendance fonctionnelle des individus suivis en rééducation et déterminer les activités humaines et/ou techniques nécessaires pour accomplir les principales activités de la vie quotidienne. Elle évalue les soins requis et mesure l'incapacité fonctionnelle. Elle comprend 18 fonctions réparties en six sous-groupes : le contrôle des sphincters, la locomotion, les transferts, les soins personnels, la communication et le comportement social. Sur l'ensemble de ces 18 items, deux sous-échelles sont individualisées, une motrice (13 critères) et une cognitive (cinq critères), mesurées sur une échelle de 1 à 7 . Les scores varient de 18 (dépendance totale) à 126 (indépendance totale). La MIF initiale ne préjuge pas du handicap séquellaire [19]. Trois groupes de patients ont été définis en fonction du score de la MIF à l'admission, groupe 1 (MIF < 60), le groupe $2(61<\mathrm{MIF}<80)$ et le groupe 3 (MIF > 80). Le groupe 1 représente les patients, dont le pronostic fonctionnel est le plus péjoratif. Le groupe 2 représente la population la plus apte à bénéficier d'une prise en charge en unité de rééducation spécialisée, avec une progression de la MIF de plus de 30 points. Le groupe 3 représente les patients qui ont conservé une assez bonne autonomie, dans les suites de l'AVC. Le taux de retour au domicile est de l'ordre de $35 \%$ pour le groupe 1 (MIF $<60$ ), de $75 \%$ pour le groupe $2(61<$ MIF $<80)$ et de $89 \%$ pour le groupe 3 (MIF > 80) [19]. Les résultats obtenus aux Charpennes pour une population âgée $(81,2$ ans) sont proches de ceux

\begin{tabular}{|c|c|c|c|}
\hline Troubles visuels & $2,3 \%$ & Surcharge pondérale & $2,3 \%$ \\
\hline Pathologies cardiaques & $18,6 \%$ & Insuffisance respiratoire & $4,6 \%$ \\
\hline Pathologies ostéoarticulaires & $4,6 \%$ & Pathologies vasculaires & $16,3 \%$ \\
\hline Troubles cognitifs & $9,2 \%$ & Dépression & $13,9 \%$ \\
\hline Pathologies neurologiques & $23,3 \%$ & Escarre & $2,3 \%$ \\
\hline Pathologies néoplasiques & $2,3 \%$ & & \\
\hline
\end{tabular}


obtenus pour une population plus jeune (70,8 ans) [19] (Tableau 1).

La progression de la MIF entre l'admission et la sortie de l'unité SSR de la filière AVC des Charpennes est de 17,3 contre 20,9 , pour une population plus jeune $(70,8$ ans) [19]. La récupération fonctionnelle ne semble pas dépendre de l'âge des patients.

\section{Personne âgée et AVC : une situation particulière}

Le devenir du patient âgé ayant présenté un AVC se pose en termes de mortalité [20], car elle est estimée à $30 \%$ à un an et environ à $50 \%$ à cinq ans, mais aussi de fonctionnalité. La cause $\mathrm{du}$ décès est souvent neurologique et dans une plus faible proportion cardiaque. Le pronostic fonctionnel des patients âgés après un AVC dépend des capacités fonctionnelles initiales et des comorbidités associées. Elles sont essentiellement cognitivocomportementales, psychiatriques avec notamment la dépression, cardiovasculaires, métaboliques et ostéoarticulaires et neurosensorielles. Sur l'année 2008, pour l'unité des Charpennes, les comorbidités sont représentées par les pathologies neurologiques $(23,3 \%)$. Ce sont essentiellement des séquelles motrices de précédents AVC, de maladie de Parkinson associées. Les pathologies cardiaques (18,6 \%) sont dominées par les troubles du rythme (fibrillation atriale), souvent mal tolérés et responsables dans un cas sur deux de décompensation cardiaque gauche. Les pathologies vasculaires $(16,3 \%)$ sont représentées par des tableaux d'ischémie aiguë du membre inférieur, nécessitant un transfert dans un service de chirurgie vasculaire, et les maladies thromboemboliques.

La fragilité, indépendamment de toute pathologie, est un état instable qui résulte d'une baisse des réserves d'adaptation entrânant un risque élevé de dépendance (multiplié par 3), d'hospitalisation (multiplié par 2), d'entrée en institution, de mortalité (multiplié par 3). Les causes sont multiples, notamment génétiques, iatrogènes et socio-environnementales. Parmi les critères de fragilité, on observe une diminution de la force de préhension, de la vitesse de marche et la perte d'activité physique [21]. La prévalence augmente avec l'âge [22] pour être supérieure à $30 \%$ au-delà de 90 ans. Il apparaît déterminant de détecter cette fragilité chez les patients âgés ayant présenté un AVC, et seules des unités avec compétences gériatriques paraissent appropriées.

\section{Complications post-AVC}

\section{Dépression}

La dépression est la manifestation psychiatrique la plus fréquente après un AVC [33]. On estime sa prévalence à $30 \%$ environ selon les études, celles-ci pouvant varier de 20 à $65 \%$ [33]. La présence d'une dépression augmente le risque de mortalité et surtout influe sur le pronostic fonction- nel [33]. La récupération fonctionnelle des patients présentant une dépression est moindre et leur qualité de vie est altérée [33]. La dépression peut survenir plusieurs mois après l'AVC, un rôle propre à la lésion pouvant être parfois évoqué. Il est important de la détecter précocement et de mettre en place une prise en charge psychologique et un traitement de type inhibiteur de la recapture de la sérotonine. Il n'est pas prouvé qu'un traitement antidépresseur soit efficace à titre préventif.

Vingt-six (39,4\%) des patients accueillis dans l'unité des Charpennes, en 2008, ont présenté une dépression dans les suites de leur AVC. Six $(9,1 \%)$ de ces patients ont nécessité une prise en charge par la psychologue et le psychomotricien. Pour ces patients, la durée moyenne de séjour est supérieure à 18,3 jours en moyenne, par rapport aux patients non dépressifs et présentant le même déficit moteur.

\section{Troubles de déglutition}

Les troubles de la déglutition sont très fréquents [34,35]. Ils peuvent se résoudre en quelques semaines, mais peuvent persister quelques mois, voire être permanents. Ils sont responsables de pneumopathies d'inhalation, augmentant la morbidité et la mortalité des patients après l'AVC [36]. Ils nécessitent une prise en charge pluridisciplinaire (ORL, orthophonistes, diététiciens, soignants, gériatres). Tout patient ayant présenté un AVC doit être rapidement exploré, afin d'adapter le type de texture alimentaire. Une analyse plus détaillée de la déglutition peut être envisagée par le biais de la vidéofluoroscopie (VFS) ou radio-cinéma. Cela permet d'évaluer la nécessité du recours aux sondes nasogastriques ou à la gastrostomie. Onze $(16,7 \%)$ patients de l'unité des Charpennes, en 2008, compte tenu de troubles de déglutition, ont nécessité la pose d'une sonde nasogastrique. La rééducation a pu être poursuivie, tout en maintenant une alimentation entérale. Cette sonde a pu être enlevée, en moyenne à 21 jours, sauf pour cinq $(7,58 \%)$ patients qui ont nécessité la pose d'une sonde de gastrostomie. Un élément important est que pour deux des cinq patients, la sonde de gastrostomie a pu être retirée au bout d'un an. La rééducation des troubles de déglutition se justifie, même à distance de l'AVC.

\section{Troubles cognitifs}

Un syndrome démentiel est fréquemment observé dans les six mois après la survenue d'un AVC [37]. L'apparition concomitante d'un syndrome démentiel et d'une lésion vasculaire n'est pas un gage de démence vasculaire pure, une lésion ischémique ou hémorragique pouvant révéler une maladie neurodégénérative [38]. Dans ces cas, alors que la maladie neurodégénérative n'est pas encore symptomatique, la lésion aiguë vient réduire brutalement la réserve 
cognitive et occasionne l'apparition des signes cliniques de défaillance. L'évolution secondairement progressive du syndrome démentiel permet alors d'évoquer un processus dégénératif sous-jacent. La progression en marches d'escaliers du syndrome démentiel est évocatrice mais inconstante, surtout en cas d'état multilacunaire et de leucoencéphalopathie. Pour évoquer une démence vasculaire, il est déterminant de pouvoir expliquer les symptômes par les lésions visibles sur l'imagerie, en particulier un syndrome sous-cortico-frontal par des lésions diffuses de la substance blanche, parfois un déficit cognitif spécifique en rapport avec une lésion stratégique [38,39]. L'IRM encéphalique est particulièrement utile pour faire l'inventaire des lésions, en particulier les séquences FLAIR pour les lésions ischémiques anciennes, les séquences de diffusion pour les lésions ischémiques récentes, les séquences $\mathrm{T} 2 *$ pour les lésions microhémorragiques, le scanner crânien étant peu sensible.

La durée moyenne de séjour à l'unité des Charpennes, en 2008, des patients atteints de troubles cognitifs dépasse de 21,4 jours celle des patients n'ayant aucun trouble cognitif. Ces patients présentent des troubles attentionnels et des difficultés à retenir les consignes de sécurité à la marche. La progression de la MIF pour ces patients ayant des troubles cognitifs est superposable aux autres patients $(17,2$ versus 17,4$)$. Le déclin cognitif ne semble pas être un facteur prédictif de la récupération fonctionnelle.

\section{SSR gériatrique AVC}

La nouvelle circulaire DHOS/DGS/DGAS $\mathrm{n}^{\circ} 108 \mathrm{du}$ 22 mars 2007, en complément de celle du 3 novembre 2003, souligne l'importance de la fluidité de la filière et surtout redéfinit l'organisation des SSR non plus en fonction de la typologie des structures mais plutôt en fonction des affections prises en charge. Initialement les soins de suite et de réadaptation étaient organisés selon le principe de segmentation " verticale » par population et/ou par prise en charge, selon trois catégories : rééducation-réadaptation fonctionnelle, soins de suite, maison de repos. Ils sont désormais présentés selon un principe de gradation « horizontale " (mode polyvalent/recours) avec en particulier des unités de SSR neurologiques. Ces nouvelles unités feront appel à des compétences spécifiques de l'équipe qui les compose.

Le bénéfice des unités neurovasculaires spécialisées a été montré sur la mortalité et l'indépendance, quel que soit l'âge du patient [40], notamment pour tous les patients étant indépendants avant l'AVC. La rééducation doit être poursuivie dans des structures différentes selon l'état du patient et la sévérité de l'AVC. Cette efficacité se mesure en particulier sur la mortalité, le degré d'autonomie et le pourcentage de retour au domicile. L'efficacité de la rééducation tient autant aux techniques spécifiques qu'à l'intensité relative de la rééducation et à l'organisation en équipe pluridisciplinaire.

\section{Quels objectifs ?}

La prise en charge de ces patients doit intégrer les soins spécifiques liés à l'AVC et la globalité de la personne âgée, en particulier la polypathologie et la fragilité potentielle. Les objectifs d'une prise en charge des patients âgés ayant présenté un AVC pourraient être principaux ou autres.

\section{Objectif principal}

- Reprise d'une autonomie à la marche, qui requiert des conditions :

- une récupération fonctionnelle suffisante des membres inférieurs ;

- des fonctions cognitives préservées ;

- un état orthopédique antérieur correct ;

- une prise en charge rapide et de qualité.

\section{Autres objectifs}

- Retour à un niveau d'autonomie « honorable »;

- récupération du contrôle sphinctérien ;

- récupération de la déglutition;

- récupération des troubles phasiques ;

- prise en charge des troubles psychiatriques et en particulier la dépression ;

- le retour au domicile, garant d'une qualité de vie.

Le contrôle du tronc en position assise, de la station debout et de la marche conditionne le retour au domicile. Certaines études montrent que la reprise de la marche peut être obtenue dans $80 \%$ des cas, la moitié des patients utilisant une aide technique (cannes, déambulateur, attelles) $[28,29]$. Cette reprise de la marche peut être maintenue à un an. Il est par ailleurs démontré que les patients ayant bénéficié d'une prise en charge dans des unités spécialisées ont un maintien, voire une progression de l'acquis fonctionnel, supérieur à ceux qui n'ont pu être admis dans des unités spécialisées [30]. La récupération de la préhension est un élément très important pour les activités de la vie quotidienne. Les taux de récupération sont variables en fonction des études, pouvant atteindre 25 à $60 \%$ [31,32]. Dans le cadre de la population âgée des Charpennes, la récupération de la préhension reste faible à $19,8 \%$. La reprise de la marche est maintenue à $85 \%$ à un an après leur sortie de l'unité.

\section{Entretenir le potentiel récupéré}

Cela ne peut être envisagé que par le développement de structures de médecine physique et de rééducation en hôpital 
de jour (HDJ) ou équipe mobile, qui représente une excellente transition entre la structure hospitalière et le domicile [5], avec un suivi par une équipe pluridisciplinaire spécialisée. Le suivi permet d'analyser la qualité du maintien à domicile, d'évaluer les besoins du patient et de sa famille. Il est envisageable de prévoir des visites à domicile avec l'ergothérapeute et l'assistante sociale. Le patient est de même suivi par le médecin rééducateur et le gériatre qui adaptent les traitements et qui s'assurent de l'équilibration des comorbidités et des différents facteurs de risques. Ainsi, cette équipe peut avoir une action de prévention et limiter les risques de réhospitalisation.

\section{Vers des unités " globales » associant soins de courte durée (SCD) et SSR}

Les Canadiens ont très récemment émis des recommandations pour la prise en charge optimale des soins de l'AVC. Ils ont défini trois types d'unités [36] :

- les « unités intégrées » d'AVC ;

- les unités d'AVC aigu ;

- les unités de réadaptation d'AVC.

Les unités intégrées d'AVC regroupent des lits de SCD et les lits de réadaptation. Ainsi, l'équipe qui compose ce type d'unité peut prendre en charge la phase aiguë de l'AVC et débuter une rééducation précoce du patient. Cette prise en charge est coordonnée par une équipe pluridisciplinaire comprenant une équipe médicale et de rééducation, ce qui facilite la prise en charge globale du patient. Ce type d'unité correspond tout à fait au profil du patient âgé. L'unité de SSR de la filière AVC des Charpennes (CHU de Lyon) tend vers ce type d'orientation. En septembre 2010, de par la composition de notre équipe, notre activité passera de dix lits de SSR gériatriques à 20 lits, dont quatre lits de $\mathrm{SCD}$ et 16 lits de SSR dédiés à l'AVC, et ce, au sein d'une même unité.

\section{Conclusion}

L'efficacité des soins spécifiques dédiés à la personne âgée victime d'un AVC, à la phase aiguë et en SSR, est observée sur le pronostic fonctionnel et le taux de retour au domicile. En unité spécialisée [41], la durée moyenne de séjour est plus faible, les complications post-AVC sont moins importantes, le taux de retour au domicile est plus important. Il apparaît nécessaire d'améliorer la filière de soins des AVC chez la personne âgée qui n'a pas toujours accès aux UNV. Certaines expériences d'UNV SSR dédiées à la personne âgée sont concluantes. Il est impératif de maintenir une équipe médicale et soignante multidisciplinaire, intervenant à la phase aiguë et en SSR, pour délivrer des soins spécialisés, prendre en charge la fragilité et la polypathologie, caractéristiques de la personne âgée. Ce type d'hospitalisation en unité « globale » peut être suivi d'une prise en charge pluridisciplinaire à l'HDJ, où la poursuite de la rééducation peut être organisée.

\section{Références}

1. Leys D, Godefroy O, Pasquier F (1998) Pronostic des accidents ischémiques cérébraux. Rev Prat 48:171-4

2. Circulaire DHOS/DGS/DGAS (2003) Relative à la prise en charge des accidents vasculaires cérébraux

3. Brissot R, Perrigot M (1997) Hémiplégie et vieillissement. ANMSR

4. Chatard S, David JS, Boisson D (2009) Étude AVC 69 : étude de cohorte prospective dans les établissements se soin du Rhône du 6 novembre 2006 au 6 juin 2007. resuval.univ-lyon1.fr/SC.pdf

5. Yelnik A, Bradaï N (2005) Rééducation du sujet âgé après accident vasculaire cérébral. Psychol Neuropsychiatr Vieil 3:157-62

6. Varenne O. 2004. La prise en charge de l'AVC. La revue du praticien, vol 5

7. Leys D, Kwiecinski H, et al (2004) Prevention European Stroke Initiative. Cerebrovasc Dis 17:15-29

8. Lo E, Pradat-Diehl P (1999) Prise en charge kinésithérapeutique de l'AVC. Neurologies 1-6

9. Rouleaud S, Dehail P (2001) La marche de l'hémiplégique. Ann Readap Med Phy 44:172-5

10. Retour au domicile des patients atteints d'AVC (2003) Anaes : stratégies et organisation

11. Daviet JC, Verdié-Kessler C, Stuit A, et al (2006) Facteurs prédictifs du devenir fonctionnel et du retour au domicile après un premier accident vasculaire cérébral hémisphérique. Ann Readap Med Phy 49(2):49-56

12. Kalra L, Evans A, Perez I, et al (2000) Alternative strategies for stroke care: a prospective randomised controlled trial. Lancet 356:894-9

13. Stroke Unit Trialist's Collaboration. (2002) Organised inpatient (stroke unit) care for stroke (Cochrane Review). In: The Cochrane Library, Issue 1. Update Software, Oxford

14. Candelise L, Gattinori M, Bersano A, et al (2007) Stroke unit care for acute stroke patients: an observational follow-up study. Lancet 369:299-305

15. Calmels P, Defay C, Yvanes-Thomas M, et al (2005) L'âge très élevé constitue-t-il un facteur pronostique du devenir après un premier accident vasculaire cérébral ? Ann Readap Med Phy 48:675-81

16. Dauphin A, Thevenon A (1997) Critères de choix des filières de soins dans la rééducation de l'hémiplégie vasculaire. Ann Readap Med Phy 40:255-64

17. Bagg S, Paris Pombo A, Hopman W (1989) Effect of age on fonctionnal outcomes after stroke rehabilitation. Stroke 20: 864-70

18. Hamilton BB, Granger CV, Sherwin F, et al (1987) A uniform national data system for medical rehabilitation system (UBS). In: Furher JM (ed) Rehabilitation outcomes: analysis and measurement. Lippinott, Baltimore, pp 137-47

19. Galin LC, Rode G, Soler-Michel P, et al (1994) Intérêt de l'étude de la mesure d'indépendance fonctionnelle (MIF) dans l'évaluation de la rééducation des sujets hémiplégiques âgés de plus de 60 ans. Ann Readap Med Phy 37:281-90 
20. Wilkinson PR, Wolfe CA, Warburton FG, et al (1997) A longterm follows-up of stroke patients. Stroke 28:507-12

21. Walston J, Mc Burnie MA, Newman A, et al (2002) Frailty and activation of the inflammation and coagulation systems with and without clinical comorbidities: results from the cardiovascular health study. Arch Intern Med 162:2333-41

22. Ahmed N, Mandel R, Mindy J, et al (2007) Frailty: an emerging geriatric syndrome. Am J Med 120:748-53

23. Lenzi CL, Altieri M, Maestrini I (2008) Post-stroke depression. Réunion commune de la SFN et de la SFNV, 8-10 octobre 2008. Rev Neurol 164:837-40

24. Falsetti P, Acciai C, Palilla R, et al (2009) Oropharyngeal dysphagia after stroke; incidence, diagnosis and clinical predictors in patients admitted to a neurorehabilitation unit. J Stroke Cerebrovasc Dis 5:329-35

25. Troubles de la déglutition et MPR. Vers une prise en charge pluridisciplinaire. Communications présentées lors du $23^{\mathrm{e}}$ Congrès national de la Sofmer à Mulhouse du 22 au 25 octobre 2008. Ann Med Phy 51:565-7

26. Vandemeulebroecke M, Laloux P, Lawson G, et al (2007) La dysphagie après un accident vasculaire cérébral : bilan et prise en charge. Louv Med 126:95-101

27. Henon H, Durieu I, Guerouaou D, et al (2001) Poststroke dementia: incidence and relationship to prestrike cognitive decline. Neurology 57:1216-22

28. Chui H (2005) Neuropathology lessons in vascular dementia. Alzheimer Dis Assoc Disord 19:45-52

29. Reed BR, Mungas DM, Kramer JH, et al (2004) Clinical and neuropsychological features in autopsy-defined vascular dementia. Clin Neuropsychol 18:63-74

30. Kaste M, Palomaki H, Sarna S (1995) Where and how should elderly stoke patients be treated? A randomized trial. Stroke 26:249-53

31. Perrigot M, Bergeco C, Fackacs C, et al (1980) Hémiplégie vasculaire : bilan et éléments du pronostic de la rééducation. Ann Readap Med Phy 2:229-41
32. Jorgensen HS, Nakayama H, Rashou HO, et al (1995) Recovery of walking function in stroke patients the Copenhagen Stoke study. Arch Phys Med Rehab 76:27-32

33. Brosbois B, Brissot R, Druais JL, et al (1980) Le devenir de patients hémiplégiques vasculaires hospitalisés au CHR de Rennes en 1974-1975. Ann Med Phy 23:261-7

34. Le Thiec F, Mane M, Guincestre JY, et al (1996) L'hémiplégie vasculaire en Basse-Normandie, mortalité et devenir fonctionnel. Ann Readap Med Phy 39:131-42

35. Enjabert M, Pelissier, Blin D, et al (1988) Classification fonctionnelle de la préhension chez l'hémiplégique adulte. Hémiplégie vasculaire de l'adulte et médecine de rééducation. Masson Ed, Paris, pp 212-23

36. Un guide pour la mise en œuvre des soins en unité AVC (2009) The Canadian Stroke Strategy. Une ressource pour la mise en œuvre des recommandations canadiennes pour les pratiques optimales de soins de l'AVC

37. Lermitte P, Lejeune P, Bercelli P, et al (2008) Améliorer la prise en charge des AVC âgés en soins de suite : étude rétrospective sur deux ans en neurologie et en soins de suite au CHD de La Roche-sur-Yon. $40^{\mathrm{es}}$ Journées annuelles, SGOC

38. Chiu KY, Phun WK, Lux KT (1992) AVC du sujet âgé. Injury 27:2183-6

39. $20^{\mathrm{e}}$ Congrès national de la Sofmer (2005) Ann Readap Med Phy 48:138-45

40. Pérennou D, El Fatimi A, Masmoudi M, et al (2005) Incidences, circonstances et conséquences des chutes chez les patients en rééducation après un premier accident vasculaire cérébral. Ann Readap Med Phy 48:138-45

41. Chabot-Henri C, Diakité A, René-Corail, et al (2006) ERMANCIA : le devenir à cinq ans d'une cohorte d'AVCi. Rev Geriatr, tome $31, n^{\circ} 6$

42. Kahlaoui K, Ansaldo AI (2009) Récupération de l'aphasie d'origine vasculaire : facteurs pronostiques et apport de la neuro-imagerie fonctionnelle. Rev Neurol 165:233-42 